\title{
Dust Evolution in Intermediate Velocity Clouds
}

\author{
Pinheiro Gonçalves, D.* \\ Canadian Institute of Theoretical Astrophysics \\ E-mail: dcpgecita.utoronto.ca
}

Martin, P.G. and Blagrave, K.

Canadian Institute of Theoretical Astrophysics

E-mail: pgmartin@cita.utoronto.ca, blagrave@cita.utoronto.ca

\section{Miville-Deschênes, M.-A.}

Institut d'Astrophysique Spatiale, Université Paris-Sud 11

E-mail: mamdeias.u-psud.fr

\begin{abstract}
We search for evidence of dust evolution in high Galactic latitude regions by looking at variations in the emissivities of dust associated with different velocity clouds. In order to do so, we spatially correlate infrared IRAS/IRIS dust maps with HI column density maps derived from 21-cm radio observations with the GBT. Our findings show that intermediate velocity clouds (IVCs or halo clouds) have a higher $60 \mu \mathrm{m} / 100 \mu \mathrm{m}$ and lower $12 \mu \mathrm{m} / 100 \mu \mathrm{m}$ color ratio when compared to dust in local low-velocity gas. This suggests that large thermal dust grains are shattered into smaller ones (VSGs) and that there is a low relative abundance of PAHs in IVCs.
\end{abstract}

The Life Cycle of Dust in the Universe: Observations, Theory, and Laboratory Experiments - LCDU 2013, 18-22 November 2013

Taipei, Taiwan

${ }^{*}$ Speaker. 


\section{Introduction}

The evolution of dust (which is a vital component in our Galaxy) through the several ISM stages is a key influence on other Galactic phenomena. Therefore, it is important to conduct studies to answer fundamental questions about the dust properties, content and its continuous processing. The latter effect is now being incorporated into theoretical dust models ([3]) to explain changes in the relative contribution of each dust population in the different dust SEDs observed in the ISM. One way to obtain information on processed dust is to look at dust associated with IVCs which are part of the Galactic fountain. These clouds are thought to be the result of dust and gas which have been processed by supernovae shocks and HII regions. Thus, one would expect there to be some shattering of large dust grains ([4]). Our focus here is to measure the infrared (IR) emissivities of dust associated with these halo clouds and compare them with their local dust counterpart in order to infer similarities or differences due to dust processing.

\section{Recipe for Data Analysis}

We start with the usual recipe for measuring the dust emissivities by performing spatial correlations. Here, we use the IR data from IRAS/IRIS survey ([6]) at 12, 25, 60 and $100 \mu \mathrm{m}$ together with single-dish 21 -cm HI radio data ( $9^{\prime}$-resolution) from the Green Bank Telescope (GBT) to study fields at high Galactic latitudes. The 800 square degrees of HI data ([1]) also have a good velocity resolution which allows for velocity component separation into low, intermediate and high velocity gas. Following [7], we have masked parts of our fields to avoid high HI column density $\left(\mathrm{N}_{\mathrm{HI}}\right)$ areas where the dust-gas correlation breaks and we have distinguished between bright and faint $\left(\left\langle\mathrm{N}_{\mathrm{HI}}\right\rangle<10^{20} \mathrm{~cm}^{-2}\right.$ ) fields. We slightly modify the correlation to include a smooth gradient in the form of a tilted plane (parameterized by the coefficients $P_{\mathrm{x}}$ and $P_{\mathrm{y}}$ ) which mostly accounts for imperfect zodiacal light removal at mid-IR wavelengths in the IRIS/IRAS data $\left(\mathrm{I}_{\lambda}\right)$. The dust emissivities $\left(\varepsilon_{\lambda}^{i}\right)$ at each wavelength $(\lambda)$ and velocity component $(i)$ are given by solving the following equation:

$$
\mathrm{I}_{\lambda}(x, y)=\sum_{i=1}^{3} \varepsilon_{\lambda}^{i} \mathrm{~N}_{\mathrm{HI}}^{i}(x, y)+a_{\lambda} P_{\mathrm{x}}(x, y)+b_{\lambda} P_{\mathrm{y}}(x, y)+d_{\lambda},
$$

where the intercept $d_{\lambda}$ represents the contribution of any residuals in the IR data which do not correlate with the HI data. We apply this methodology to all our 14 GBT fields and obtain emissivities of dust associated with different velocity clouds at all 4 of the IRAS wavelengths.

\section{Results}

We combine our measurements with the PLANCK emissivities found by [7] for the same fields to build SEDs from the mid-IR to the sub-millimeter regime (an example is shown in the left plot of Figure 1). Uncertainties were estimated using a Monte-Carlo method as described in the work of [7]. We compare the obtained IR emissivities for the IVCs with what would be expected from the DustEM diffuse ISM dust model ([2]). This model was determined by fitting dust emissivities in the whole sky at the same wavelengths but without any velocity component separation. At $12 \mu \mathrm{m}$, the value of $\varepsilon_{\mathrm{IVC}}$ is lower suggesting a depletion of grains emitting at $12 \mu \mathrm{m}$ (PAHs, Polycyclic 

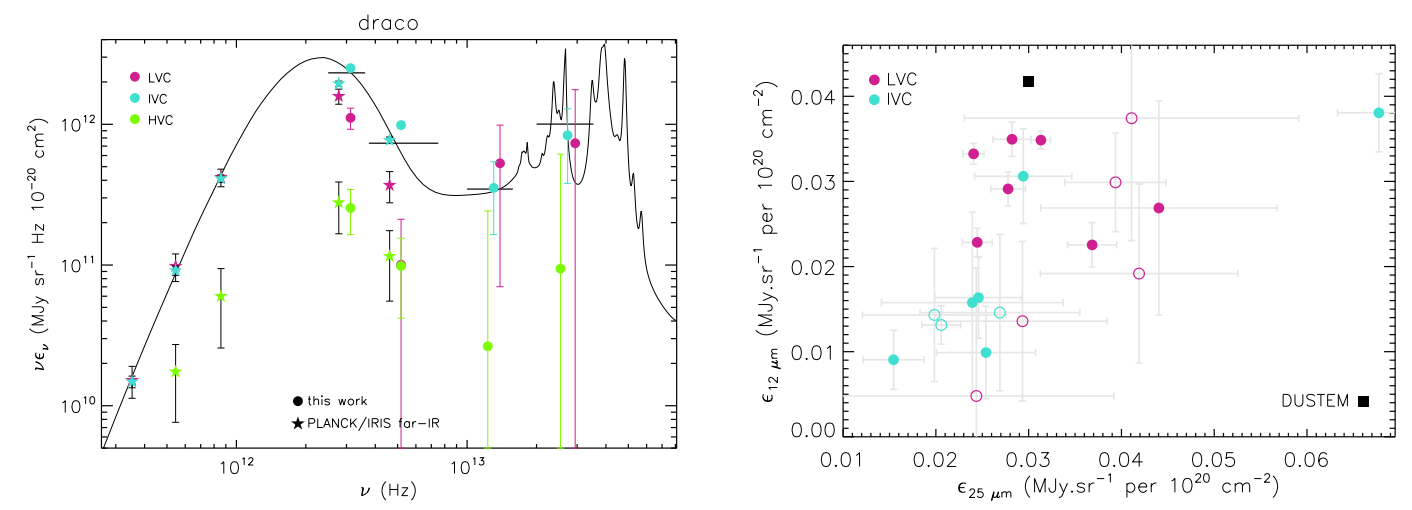

Figure 1: Left: SED from the mid-IR to the submillimeter for draco (wavelength increasing from right to left). Violet, blue and green circles are the estimates for the local, intermediate and high-velocity dust emissivities. Star symbols locate the results from the work of [7]. Overplotted in black is the standard dust model for the diffuse ISM as predicted by the DustEM code and it works as a common reference curve. The black horizontal lines at the IRAS wavelengths show the SED value of the dust model as it would be seen through the IRAS filters. Right: Diagrams of the 12 and 25 $\mu \mathrm{m}$ dust emissivities. Filled circles represent bright fields while open circles are for faint fields. Local dust emissivities are presented in violet, while IVCs are in blue as indicated in the plots. The black square marks the corresponding IRAS colors of the standard DustEM diffuse ISM dust model.

Aromatic Hydrocarbons). On the other hand, the $25 \mu \mathrm{m}$ emissivities are comparable (or only slightly lower) to what would be expected from the model, although its interpretation is not simple, due to the contributions of both dust populations, the PAHs and VSGs at this wavelength. To round out our analysis, we have extended our comparison to the 60 and $100 \mu \mathrm{m}$ emissivities. At $60 \mu \mathrm{m}$, IVCs show greater emissivities than local gas (as also seen in the previous work of [7]) which is a sign of a larger VSG population, possibly indicating that there has been some processing of the larger thermal grains into smaller ones. At $100 \mu \mathrm{m}$, emissivities of dust associated with low and intermediate velocities are comparable. The same conclusions can be drawn from the slightly more informative trends in the color ratio. The right plot of Figure 1 shows the correlation between the two mid-IR wavelengths, 12 and $25 \mu \mathrm{m}$. Again, IVCs display lower mid-IR colors than LVCs. Although the $25 \mu \mathrm{m}$ emissivity value seems comparable to the typical DustEM diffuse ISM dust, at $12 \mu \mathrm{m}$ its value is lower by at least a factor of 2 for most fields. The higher $60 \mu \mathrm{m}$ for IVCs is also seen in the color correlations although not displayed here. A comparison of color ratios shows that IVCs have a 1.4 times lower $\mathrm{R}_{12 / 100}$ and a 1.6 higher $\mathrm{R}_{60 / 100}$ than dust in LVCs.

Furthermore, we can investigate how much we need to change the relative contributions of each dust population in order to match the observed IVC SEDs. Figure 2 shows the same trends in the separation of IVC and LVC colors, but also includes overlays indicating the effect on the respective emissivities from changes in the DustEM model parameters. This preliminary comparison shows that IVC dust emissivities cannot simply be explained by varying the strength of the interstellar radiation field (ISRF); different relative abundances of PAHs and VSGs are needed.

\section{Summary}

We have studied the dust content of IVCs using IRAS/IRIS infrared and 21-cm radio HI GBT data (Pinheiro Gonçalves et al., in prep). When compared to local dust, IVCs show low $12 \mu \mathrm{m}$ 


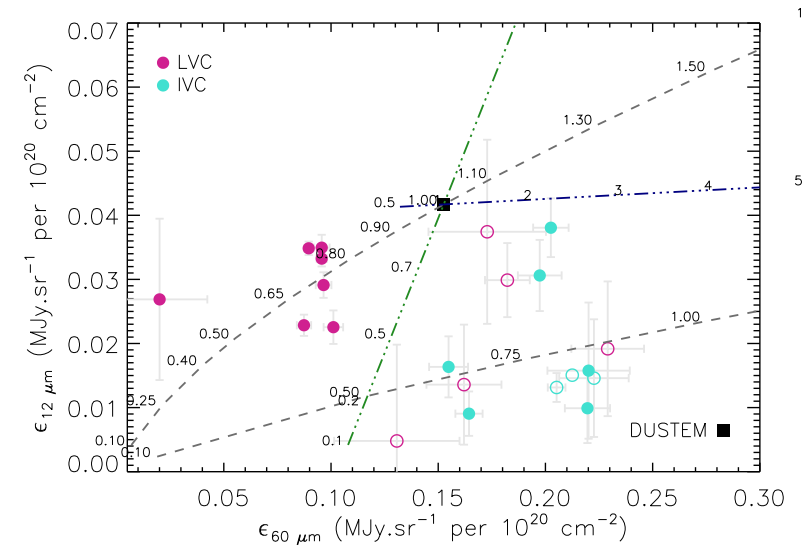

Figure 2: Diagrams of the 12 and $60 \mu \mathrm{m}$ dust emissivities. Local dust emissivities are presented in violet, while IVCs are in blue as indicated in the plots. The black square marks the corresponding IRAS colors of the standard DustEM diffuse ISM dust model. Dashed lines show the corresponding emissivities with varying ISRF strength while dot-dashed lines show the effect on the dust emissivities by varying the relative abundances of PAHs and VSGs (green) and VSGs only (blue). The lower dashed line, besides varying the ISRF strength also has 4 times of relative abundance of VSGs and half the relative abundance of PAHs when compared to the standard DustEM diffuse ISM dust model.

emissivities which suggests a depletion in the abundance of PAHs and high $60 \mu \mathrm{m}$ which can be interpreted as shattering of larger thermal grains into VSGs. Both results point towards dust processing, probably via shocks that can destroy and/or alter the size of certain dust populations, as suggested in the theoretical works of [5] and [4].

\section{References}

[1] A. I. Boothroyd, K. Blagrave, F. J. Lockman, P. G. Martin, D. Pinheiro Gonçalves, and S. Srikanth. Accurate galactic 21-cm H I measurements with the NRAO Green Bank Telescope. Astronomy and Astrophysics, 536:A81, December 2011.

[2] M. Compiègne, L. Verstraete, A. Jones, J.-P. Bernard, F. Boulanger, N. Flagey, J. Le Bourlot, D. Paradis, and N. Ysard. The global dust SED: tracing the nature and evolution of dust with DustEM. Astronomy and Astrophysics, 525:A103, January 2011.

[3] A. P. Jones, L. Fanciullo, M. Köhler, L. Verstraete, V. Guillet, M. Bocchio, and N. Ysard. The evolution of amorphous hydrocarbons in the ISM: dust modelling from a new vantage point. Astronomy and Astrophysics, 558:A62, October 2013.

[4] A. P. Jones, A. G. G. M. Tielens, and D. J. Hollenbach. Grain Shattering in Shocks: The Interstellar Grain Size Distribution. Astrophysical Journal, 469:740, October 1996.

[5] E. R. Micelotta, A. P. Jones, and A. G. G. M. Tielens. Polycyclic aromatic hydrocarbon processing in interstellar shocks. Astronomy and Astrophysics, 510:A36, February 2010.

[6] M.-A. Miville-Deschênes and G. Lagache. IRIS: A New Generation of IRAS Maps. Astrophysical Journal, Supplement, 157:302-323, April 2005.

[7] Planck Collaboration, A. Abergel, P. A. R. Ade, N. Aghanim, M. Arnaud, M. Ashdown, J. Aumont, C. Baccigalupi, A. Balbi, A. J. Banday, and et al. Planck early results. XXIV. Dust in the diffuse interstellar medium and the Galactic halo. Astronomy and Astrophysics, 536:A24, December 2011. 